\title{
Presentación oral como evaluación comunicacional del médico residente de cirugía general
}

\author{
Eduardo B. Arribalzaga
}

Objetivo. Describir la presentación oral de un caso clínico-quirúrgico en la reunión de un servicio asistencial y el desempeño comunicacional del médico residente.

Sujetos y métodos. Estudio observacional descriptivo longitudinal prospectivo, con análisis transversal en diferentes momentos de la presentación de casos clínicos. Participaron 10 médicos residentes de cirugía general en su cuarto año de residencia en un mismo hospital universitario entre el 30 de marzo de 2016 y el 30 de septiembre de 2017. El caso clínico se presentaba oralmente en una reunión general semanal del Servicio de Cirugía General con sólo dos casos clínicos por reunión, sin posibilidad de debate por el médico residente. La evaluación se basaba en la calificación de la presentación oral del caso clínico con seis criterios y cuya verificación de calidad de cada ítem oscilaba entre 1 (deficiente) y 10 (excelente). Hubo un solo evaluador.

Resultados. De las 76 presentaciones realizadas, la nota calificadora media obtenida por los médicos residentes fue de 8,2 , con claros signos de mejor desempeño al presentar más casos clínicos. Con las notas observadas se creó un listado calificador del desempeño de los médicos residentes en sus distintas actuaciones. La fiabilidad como instrumento de valor fue aceptable $(0,76)$.

Conclusión. La presentación oral de un caso clínico por médicos residentes de cirugía es un instrumento de evaluación comunicacional con un elevado grado de validez y fiabilidad debido a los criterios preestablecidos para una evaluación objetiva y efectiva. Mayor cantidad de presentaciones, mayor experiencia y mejor desempeño.

Palabras clave. Caso clínico. Evaluación. Presentación oral. Residencia quirúrgica.

\section{Oral presentation as communication evaluation of surgery medical resident}

Aim. To describe the oral presentation of a clinical case in a meeting and medical resident communication performance.

Subjects and methods. Prospective observational study descriptive longitudinal, cross-sectional analysis in different times of clinical cases oral presentation. Ten general surgery resident physicians take part in his 4th year of residence at a university hospital between March 30, 2016 and September 30, 2017. The clinical case was showed in the weekly general meeting of the General Surgery service with oral presentation exclusively two clinical cases were made. Evaluation of medical residents was based on the qualification of the oral presentation of the clinical case through 6 criteria and whose quality of each item check ranged between 1 (poor) and 10 (excellent). There was only one evaluator.

Results. From 76 presentations, it is observed that the note obtained by medical residents average oral presentations of clinical cases rating was 8.2, with clear signs of improved performance by presenting more clinical cases. Observed notes created a ranking of the performance of medical residents. Reliability as an instrument of value was acceptable (0.76).

Conclusion. The oral presentation of a clinical case for surgery medical resident in a weekly meeting as its performance assessment tool has a high degree of validity and reliability due to objectively and effective criteria evaluation preestablished. More oral presentations, more experience and better performance.

Key words. Clinical case. Evaluation. Oral presentation. Surgery medical residence.
Departamento de Cirugía. Hospital de Clínicas. Universidad de Buenos Aires. Buenos Aires, Argentina.

Correspondencia:

Dr. Eduardo B. Arribalzaga.

Departamento de Cirugía. Hospital de Clínicas. Universidad de Buenos Aires. Avda. Córdoba, 2351. CP 1122. Buenos Aires, Argentina.

E-mail:

piedralta@gmail.com

Recibido:

14.08.18.

Aceptado:

27.09.18.

Conflicto de intereses: No declarado.

Competing interests: None declared.

(c) 2018 FEM 


\section{Introducción}

El proceso de enseñanza-aprendizaje de la medicina, con un importante componente práctico (técnicas como la comunicación [1], el trabajo en equipo [2] o la adquisición de conocimientos durante la residencia médica o las carreras de especialización), es permanentemente criticado por su desigual efectividad.

Se han propuesto diferentes metodologías para mejorar este aprendizaje teniendo en cuenta el estilo de aprendizaje [3] o la forma de evaluación: resolución de problemas, evaluación clínica objetiva estructurada, utilización de portafolios, simulación médica [4] o exámenes de evaluación [5]. Una de las formas poco exploradas como método es la presentación oral de casos clínicos, en algunos lugares conocido como 'ateneo clínico' [6], que permite integrar habilidades comunicativas básicas e información clínica trascendente del paciente según una planificación previa definida. Por tanto, la competencia profesional es un conjunto de conocimientos, destrezas y actitudes, definidas en la acción, no desligadas del contexto donde se desarrollan (en este caso, la residencia médica quirúrgica) y que se cultivan mediante la experiencia real con el desarrollo de una responsabilidad progresiva e independiente.

La evaluación de esos aprendizajes en la presentación oral de un caso clínico puede interpretarse de diferente manera por distintos médicos observadores sin reflejar la utilidad real de esta herramienta de aprendizaje para la atención sanitaria personalizada. Al hacer énfasis en la evaluación, esta investigación tiene como objetivo describir la presentación oral de caso clínico como otra forma cotidiana de evaluación del desempeño comunicacional de un médico residente y considerar si éste era el esperado a medida que progresa durante el desarrollo de su programa de residencia.

\section{Sujetos y métodos}

Se planteó un estudio observacional descriptivo longitudinal prospectivo, con análisis transversal en diferentes momentos de la presentación de casos clínicos. Participaron médicos residentes de cirugía general en su cuarto año de residencia en un mismo hospital universitario, que no habían presentado anteriormente, pero seguían un esquema preestablecido; el período considerado transcurrió entre el 30 de marzo de 2016 y el 30 de septiembre de 2017. El caso clínico se mostraba en la reunión general semanal del Servicio de Cirugía General y concu- rrían la totalidad de los médicos residentes del programa de residencia, los médicos de planta y los jefes de las distintas especialidades quirúrgicas que pertenecen a dicho servicio. La presentación de un caso clínico, ya resuelto, comprendía una exposición (de cirugía general, cirugía torácica, cirugía gastroenterológica, cirugía vascular, cirugía oncológica o cirugía plástica) donde se analizaban los pormenores clínicos entre el médico residente comunicador y los asistentes a la reunión del servicio asistencial. También se detallaba la participación de especialistas como médicos radiólogos o anatomopatólogos que debatían el caso clínico presentado. Al finalizar cada caso clínico con su resolución quirúrgica y el seguimiento postoperatorio inmediato (30 días), se debatía acerca de las decisiones ya efectuadas; no había posibilidad de debate por parte del médico residente, sino que éste sólo comunicaba lo ya hecho y se analizaba con una referencia bibliográfica de los últimos cinco años para fundamentar la conducta terapéutica seguida. Se presentaban exclusivamente dos casos clínicos por reunión. Los temas de las patologías eran los más representativos o complejos de las diferentes especialidades quirúrgicas.

Se instruyó previamente cómo debía ser una presentación clínica adecuada según distintos pasos a seguir (Tabla I).

Cada uno de los médicos residentes evaluados fue designado con un número para preservar su anonimato. El único evaluador fue el autor de este trabajo, quien además realizó el análisis comparativo de todas las presentaciones orales en los distintos ateneos del período examinado. Los médicos residentes presentaron casos clínicos como mínimo en cinco ateneos diferentes, con un máximo de $10 . \mathrm{Al}$ terminar la reunión, se ofrecía a cada médico residente una devolución de su evaluación acerca de su desempeño comunicacional para corregir fallos.

La evaluación de la presentación del caso clínico en el ateneo se realizaba con seis criterios y una verificación de calidad de cada ítem mediante una escala de valoración numérica que oscilaba entre 1 (deficiente) y 10 (excelente): la calificación máxima a obtener era de 60 puntos, con un mínimo de 36 puntos para considerar aprobada la evaluación. Ello sirvió para comparar la validez real de esta herramienta de evaluación en una presentación oral. Los seis criterios evaluables eran:

C1. Claridad de expresión: lenguaje claro, conciso, remarca ideas claras.

C2. Capacidad de síntesis: identifica objetivos de forma ordenada y coherente.

C3. Cantidad adecuada de imágenes: esquemas, imágenes, videos, fotos. 
C4. Calidad adecuada de imágenes: diseño adecuado, dibujos y gráficos con información relevante.

C5. Secuencia lógica de presentación: tema, su importancia, resultados, conclusiones.

C6. Coherencia de lenguajes verbal y gestual: gestos sin abusar para resaltar, mantener la atención de la audiencia.

Se analizó con pruebas estadísticas ( $t$ de Student), una vez establecido el tamaño muestral necesario para variables continuas de una población finita, mediante el programa estadístico SPSS v. 23, con aceptación de una diferencia significativa para $p \leq 0,05$.

La fiabilidad (o consistencia) en la evaluación de la presentación de un caso clínico se midió con el coeficiente $\alpha$ de Cronbach [7], con el procedimiento de la matriz de correlación de los ítems, que varía de 0 a 1.

\section{Resultados}

El mínimo determinado exigible del tamaño muestral para una población finita fue de seis participantes, con un número real comprobado de 10 médicos residentes y un análisis total de 76 presentaciones de casos clínicos en 38 reuniones.

El resultado de las observaciones de las presentaciones orales en las reuniones se detalla en la tabla II. Se comprueba cómo, al tener un mayor número de presentaciones, la evaluación de su desempeño comunicacional, medida según los criterios evaluables, demostraba una evolución y mejoría.

En la tabla III se observan los promedios del total de presentaciones de casos clínicos por cada médico residente según el criterio analizado y la media observada en cada criterio. De las 76 presentaciones realizadas, la nota general media de las presentaciones de casos obtenida por los médicos residentes fue de 8,2 , coincidente con la nota calificadora de los criterios evaluados (también 8,2). A medida que hacían más presentaciones orales, mejoraban su calificación.

Es de notar además que, con las notas observadas, se creó una escala clasificadora con el fin de establecer un listado del desempeño de los médicos residentes en sus distintas actuaciones en las reuniones: el médico residente identificado con el n. ${ }^{\circ} 6$ fue quien obtuvo el mejor promedio general de todas las presentaciones (Tabla IV).

Hubo dos médicos residentes (n. 3 y n. ${ }^{\circ}$ ) que presentaron en 10 oportunidades, dos residentes (n. 8 y n. ${ }^{\circ}$ 9) presentaron en cinco reuniones; dos residentes (n. 2 y n. ${ }^{\circ} 5$ ) con nueve presentaciones;
Tabla I. Guía para la confección de la presentación clínica.

1. Anticipe el tema a considerar en el caso clínico

2. No es necesario describir datos semiológicos de los sistemas que no tienen relación con el tema que se está desarrollando

3. Trate de dar una utilidad a toda la información que presente con iconografía relevante

4. Describa conceptualmente alguno de los tratamientos quirúrgicos porque el 'criterio quirúrgico' es importante en la evaluación y la nota

5. Trate de describir el postoperatorio y, si se trata de un cáncer o una patología que necesita un seguimiento prolongado, cada cuánto tiempo y cómo se controla al paciente

6. Referencia/s bibliográfica/s que fundamente/ $\mathrm{n}$ decisiones

Modelo de presentación de caso clínico:

1. Datos del paciente

2. Motivo de ingreso (o consulta)

3. Enfermedad actual

4. Antecedentes generales

5. Examen físico

6. Sospecha diagnóstica y diagnósticos diferenciales

7. Metodología diagnóstica a

8. Tratamiento ${ }^{a}$

9. Seguimiento ${ }^{a}$

Criterios de fallo (puntos que justifican una presentación equivocada):

1. Los datos de la enfermedad actual y el examen físico no son suficientes para elaborar el diagnóstico

2. Tratamiento quirúrgico conceptual incompleto o incorrecto

a Pueden usarse algoritmos

dos residentes $\left(\mathrm{n} .^{\circ} 6\right.$ y n. $\left.{ }^{\circ} 7\right)$ con siete presentaciones; un residente $\left(n .^{\circ} 1\right)$ con ocho presentaciones, y un residente $\left(n .^{\circ} 10\right)$ con seis presentaciones.

Los promedios de calificación obtenidos por los distintos médicos residentes durante las diversas presentaciones, analizados mediante la prueba $t$ de Student, no reflejaron diferencias estadísticamente significativas entre los 10 médicos residentes con los seis criterios evaluadores.

La prueba $\alpha$ de Cronbach de validación de esta técnica evaluadora determinó un valor de 0,76 (aceptable).

\section{Discusión}

El lugar y las circunstancias para la formación de competencias, en este caso clínicas y quirúrgicas, y su posterior evaluación no siempre son los ideales y varían entre distintos centros asistenciales, por lo 
Tabla II. Observaciones registradas $(n=76)$.

Cri- Ateneo Ateneo Ateneo Ateneo Ateneo Ateneo Ateneo Ateneo Ateneo Ateneo $\begin{array}{lllllllllll}\text { terio } & 1 & 2 & 3 & 4 & 5 & 6 & 7 & 8 & 9 & 10\end{array}$

\begin{tabular}{lllllllllll}
$\mathrm{C} 1$ & 6 & 7 & 8 & 8 & 7 & 9 & 9 & 9 \\
\hline $\mathrm{C2}$ & 7 & 7 & 8 & 7 & 7 & 8 & 9 & 9 \\
\hline
\end{tabular}

\begin{tabular}{|c|c|c|c|c|c|c|c|c|c|c|}
\hline \multirow{4}{*}{$\begin{array}{l}\text { Resi- } \\
\text { dente } 1\end{array}$} & C3 & 8 & 8 & 8 & 8 & 9 & 9 & 9 & \multicolumn{2}{|l|}{9} \\
\hline & C4 & 8 & 8 & 8 & 7 & 6 & 9 & 9 & 9 & \\
\hline & C5 & 7 & 7 & 8 & 8 & 7 & 8 & 9 & 9 & \\
\hline & C6 & 7 & 8 & 8 & 8 & 8 & 9 & 9 & 8 & \\
\hline & C1 & 8 & 8 & 8 & 8 & 7 & 9 & 9 & 9 & 9 \\
\hline & $\mathrm{C} 2$ & 8 & 8 & 8 & 7 & 9 & 8 & 9 & 9 & 9 \\
\hline
\end{tabular}

$\begin{array}{lllllllllll}\text { Resi- } & C 3 & 8 & 8 & 8 & 8 & 8 & 9 & 9 & 9 & 9\end{array}$

$\begin{array}{llllllllllll}\text { dente } 2 & \text { C4 } & 7 & 8 & 8 & 8 & 8 & 9 & 8 & 9 & 9\end{array}$

\begin{tabular}{llllllllll} 
C4 & 7 & 8 & 8 & 8 & 8 & 9 & 8 & 9 & 9 \\
\hline C5 & 8 & 8 & 7 & 8 & 8 & 9 & 9 & 9 & 8 \\
\hline
\end{tabular}

\begin{tabular}{|c|c|c|c|c|c|c|c|c|c|c|c|}
\hline & C6 & 8 & 8 & 7 & 8 & 8 & 9 & 8 & 8 & 9 & \\
\hline \multirow{6}{*}{$\begin{array}{l}\text { Resi- } \\
\text { dente } 3\end{array}$} & C1 & 7 & 7 & 7 & 7 & 8 & 8 & 8 & 9 & 9 & 8 \\
\hline & $\mathrm{C} 2$ & 7 & 7 & 7 & 8 & 8 & 8 & 8 & 8 & 9 & 8 \\
\hline & C3 & 7 & 8 & 7 & 8 & 8 & 8 & 6 & 8 & 9 & 8 \\
\hline & C4 & 8 & 7 & 7 & 8 & 8 & 8 & 6 & 9 & 9 & 9 \\
\hline & C5 & 6 & 7 & 7 & 8 & 8 & 7 & 7 & 8 & 8 & 8 \\
\hline & C6 & 7 & 7 & 7 & 8 & 8 & 8 & 7 & 7 & 9 & 8 \\
\hline \multirow{6}{*}{$\begin{array}{l}\text { Resi- } \\
\text { dente } 4\end{array}$} & $\mathrm{C} 1$ & 6 & 7 & 7 & 7 & 7 & 7 & 8 & 8 & 8 & 8 \\
\hline & $\mathrm{C} 2$ & 7 & 7 & 8 & 8 & 8 & 8 & 8 & 8 & 9 & 9 \\
\hline & C3 & 7 & 7 & 8 & 8 & 7 & 8 & 8 & 8 & 8 & 9 \\
\hline & C4 & 7 & 7 & 7 & 8 & 7 & 8 & 8 & 8 & 8 & 9 \\
\hline & $\mathrm{C} 5$ & 7 & 8 & 8 & 8 & 8 & 8 & 8 & 8 & 8 & 8 \\
\hline & C6 & 8 & 8 & 8 & 8 & 8 & 8 & 8 & 8 & 7 & 8 \\
\hline \multirow{6}{*}{$\begin{array}{l}\text { Resi- } \\
\text { dente } 5\end{array}$} & C1 & 8 & 8 & 8 & 8 & 8 & 8 & 9 & 9 & 9 & \\
\hline & $\mathrm{C} 2$ & 8 & 7 & 8 & 8 & 8 & 9 & 8 & 9 & 8 & \\
\hline & C3 & 7 & 7 & 8 & 8 & 6 & 9 & 9 & 9 & 9 & \\
\hline & C4 & 8 & 7 & 8 & 8 & 8 & 9 & 9 & 9 & 9 & \\
\hline & C5 & 8 & 8 & 8 & 8 & 7 & 8 & 8 & 8 & 9 & \\
\hline & C6 & 8 & 7 & 8 & 8 & 6 & 9 & 7 & 8 & 8 & \\
\hline
\end{tabular}

cual merece la pena realizar una investigación sobre el terreno para determinar si una herramienta de evaluación es pertinente para diferenciar desempeños durante un complejo proceso de enseñanzaaprendizaje como es un programa de residencia quirúrgica.

Una evaluación tiene una finalidad formativa cuando su objetivo principal consiste en proporcionar a los médicos residentes una retroalimentación sobre su aprendizaje y facilitar de esta manera una reflexión sobre su progreso en el proceso educativo. Por consiguiente, dentro del difícil proceso de la planificación educativa, una de las herramientas a usar para evitar la subjetividad del docente evaluador es la calificación de la presentación de un caso clínico en la reunión de un servicio asistencial mediante criterios específicos que permitan medir la pericia comunicacional de un médico residente.

Uno de los objetivos a valorar de las competencias técnicas, en este caso comunicacional, puede ser:

- Capacidad de realizar una presentación con una descripción sucinta de la historia clínica, con los principales hallazgos del examen físico.

- Descripción de los procedimientos diagnósticos complementarios correspondientes ya realizados.

- Establecer si el tratamiento quirúrgico fue el más conveniente, con su inmediato seguimiento posterior.

Dentro del repertorio de utilidades que ayudan a evaluar y mejorar las actividades prácticas de los médicos residentes se encuentra la presentación de casos clínicos en una reunión con miembros de un servicio asistencial, que por su diseño y conducción debe empezar a considerarse como una de las respuestas más satisfactorias para evaluar el cuidado médico real [8], ya que se debaten conductas diagnósticas y terapéuticas ya consumadas que demuestran el resultado final de una toma de decisión y se dictamina si se eligió la más adecuada.

Una presentación oral de caso clínico es un instrumento de evaluación basado en una escala cuantitativa o cualitativa asociada a criterios preestablecidos que miden las acciones de los médicos residentes sobre aspectos comunicacionales que deben evaluarse. Hay evaluaciones holísticas (evaluación global del aprendizaje o competencia) y analíticas (focalizan en un área concreta de aprendizaje) [9]. Se analizan casos clínicos concretos a la luz de evidencias teóricas y prácticas que convierten la presentación en un instrumento valioso para la formación médica.

Sin embargo, el uso de la presentación oral de caso clínico como evaluación no tiene muchos an- 
tecedentes en la literatura científica y la presente investigación apunta a describir, valorar y comparar su utilidad real. Se desarrolló este método de evaluación debido a la existencia real de tener pacientes con un historial clínico quirúrgico definido en un momento determinado, con la decisión de su intervención quirúrgica y las consecuencias de ésta.

Además, permitió establecer un listado clasificatorio acerca del desempeño comunicacional de los médicos residentes y destacar al mejor, ver la evolución durante su residencia a medida que realizaba nuevas presentaciones y valorar de esta forma su progresiva mejora de competencia. Podría objetarse que el único docente evaluador se basaba exclusivamente en su propia experiencia profesional individual, pero al ajustarse a un patrón de criterios evaluables bien definidos, la rigidez del sistema sólo permitió definir si se cumplían o no los criterios establecidos, su nivel de calidad (rango entre deficiente o excelente) y, consecuentemente, dejar de lado el factor subjetivo del evaluador. Puede argumentarse que la presentación del caso clínico no distingue el valor real de una evaluación, pero sólo hay que atenerse a describir el desempeño que efectivamente se cumplió.

Al definir los criterios a medir se ponderan los logros alcanzados que permiten comprender si se consiguieron las competencias técnicas establecidas en el programa educativo. Así surge que el criterio 1 (claridad de expresión) mostraba una mayor nota calificadora cuando el médico residente había participado al menos en cinco presentaciones; el criterio 2 (capacidad de síntesis) mostraba una mejor nota en algunos residentes $\left(n .^{\circ} 6, n .^{\circ} 7, n .^{\circ} 9 y\right.$ n. $\left.{ }^{\circ} 10\right)$ al tomar parte en, por lo menos, dos ateneos y expresaba un mejor rendimiento individual. Los criterios 3 y 4 analizaban la forma de presentación de los casos clínicos mediante la calidad y cantidad adecuada de imágenes que reflejaban el cuidado puesto en la iconografía a presentar desde el primer ateneo en el cual participaban. A medida que contribuían en más presentaciones, las notas calificadoras mejoraban, excepto en tres residentes (n. ${ }^{\circ} 6$, $\mathrm{n} .{ }^{\circ} 7$ y n. $\left.{ }^{\circ} 9\right)$, que siempre presentaron notas cercanas a la excelencia desde el primer ateneo y, aun así, las mejoraban. Al examinar los criterios 5 (secuencia lógica de presentación) y 6 (coherencia de los lenguajes verbal y gestual) se advertía que la totalidad de los médicos residentes mejoraban cuali/ cuantitativamente a medida que presentaban más casos en más ateneos, es decir, las habilidades evaluables demostraban un mejor desempeño a medida que se ganaba experiencia en presentar casos clínicos en una reunión donde siempre estaban ex-
Tabla II. Observaciones registradas ( $n=76)$ (cont.).

\begin{tabular}{|c|c|c|c|c|c|c|c|c|c|c|c|}
\hline & $\begin{array}{l}\text { Cri- } \\
\text { terio }\end{array}$ & $\begin{array}{c}\text { Ateneo } \\
1\end{array}$ & $\begin{array}{c}\text { Ateneo } \\
2\end{array}$ & $\begin{array}{c}\text { Ateneo } \\
3\end{array}$ & $\begin{array}{c}\text { Ateneo } \\
4\end{array}$ & $\begin{array}{c}\text { Ateneo } \\
5\end{array}$ & $\begin{array}{c}\text { Ateneo } \\
6\end{array}$ & $\begin{array}{c}\text { Ateneo } \\
7\end{array}$ & $\begin{array}{c}\text { Ateneo } \\
8\end{array}$ & $\begin{array}{c}\text { Ateneo } \\
9\end{array}$ & $\begin{array}{c}\text { Ateneo } \\
10\end{array}$ \\
\hline \multirow{6}{*}{$\begin{array}{l}\text { Resi- } \\
\text { dente } 6\end{array}$} & $\mathrm{C} 1$ & 9 & 9 & 9 & 9 & 9 & 9 & 9 & & & \\
\hline & $\mathrm{C} 2$ & 8 & 9 & 9 & 8 & 9 & 9 & 9 & & & \\
\hline & C3 & 9 & 9 & 9 & 9 & 10 & 10 & 10 & & & \\
\hline & C4 & 9 & 9 & 9 & 9 & 10 & 10 & 10 & & & \\
\hline & $\mathrm{C} 5$ & 8 & 9 & 9 & 8 & 9 & 9 & 10 & & & \\
\hline & C6 & 8 & 8 & 9 & 8 & 9 & 9 & 10 & & & \\
\hline \multirow{6}{*}{$\begin{array}{l}\text { Resi- } \\
\text { dente } 7\end{array}$} & $\mathrm{C} 1$ & 9 & 9 & 9 & 9 & 9 & 9 & 9 & & & \\
\hline & C2 & 9 & 9 & 9 & 9 & 9 & 8 & 9 & & & \\
\hline & C3 & 9 & 9 & 9 & 9 & 9 & 10 & 10 & & & \\
\hline & C4 & 9 & 9 & 8 & 9 & 7 & 10 & 9 & & & \\
\hline & $\mathrm{C} 5$ & 9 & 9 & 8 & 9 & 8 & 9 & 9 & & & \\
\hline & C6 & 9 & 9 & 8 & 8 & 9 & 9 & 10 & & & \\
\hline \multirow{6}{*}{$\begin{array}{l}\text { Resi- } \\
\text { dente } 8\end{array}$} & $\mathrm{C} 1$ & 8 & 8 & 9 & 8 & 7 & & & & & \\
\hline & $\mathrm{C} 2$ & 8 & 8 & 9 & 8 & 8 & & & & & \\
\hline & C3 & 7 & 9 & 9 & 9 & 9 & & & & & \\
\hline & C4 & 7 & 8 & 8 & 8 & 8 & & & & & \\
\hline & $\mathrm{C} 5$ & 8 & 9 & 9 & 9 & 8 & & & & & \\
\hline & C6 & 8 & 7 & 7 & 9 & 8 & & & & & \\
\hline \multirow{6}{*}{$\begin{array}{l}\text { Resi- } \\
\text { dente } 9\end{array}$} & $\mathrm{C} 1$ & 7 & 8 & 9 & 6 & 9 & & & & & \\
\hline & $\mathrm{C} 2$ & 7 & 9 & 9 & 9 & 9 & & & & & \\
\hline & C3 & 9 & 9 & 9 & 8 & 10 & & & & & \\
\hline & C4 & 9 & 9 & 8 & 8 & 10 & & & & & \\
\hline & $\mathrm{C} 5$ & 9 & 8 & 9 & 9 & 9 & & & & & \\
\hline & C6 & 8 & 8 & 9 & 8 & 10 & & & & & \\
\hline \multirow{6}{*}{$\begin{array}{l}\text { Resi- } \\
\text { dente } 10\end{array}$} & $\mathrm{C} 1$ & 8 & 8 & 8 & 9 & 8 & 8 & & & & \\
\hline & $\mathrm{C} 2$ & 8 & 9 & 9 & 8 & 7 & 9 & & & & \\
\hline & C3 & 9 & 9 & 8 & 8 & 7 & 9 & & & & \\
\hline & C4 & 8 & 9 & 8 & 8 & 7 & 9 & & & & \\
\hline & $\mathrm{C} 5$ & 8 & 9 & 9 & 8 & 8 & 9 & & & & \\
\hline & C6 & 8 & 8 & 8 & 8 & 7 & 8 & & & & \\
\hline
\end{tabular}


Tabla III. Desempeños comunicacionales observados.

\begin{tabular}{|c|c|c|c|c|c|c|c|c|c|c|c|}
\hline & $\begin{array}{c}\text { Residente } \\
1\end{array}$ & $\begin{array}{c}\text { Residente } \\
2\end{array}$ & $\begin{array}{c}\text { Residente } \\
3\end{array}$ & $\begin{array}{c}\text { Residente } \\
4\end{array}$ & $\begin{array}{c}\text { Residente } \\
5\end{array}$ & $\begin{array}{c}\text { Residente } \\
6\end{array}$ & $\begin{array}{c}\text { Residente } \\
7\end{array}$ & $\begin{array}{c}\text { Residente } \\
8\end{array}$ & $\begin{array}{c}\text { Residente } \\
9\end{array}$ & $\begin{array}{c}\text { Residente } \\
10\end{array}$ & $\begin{array}{l}\text { Promedio } \\
\text { del criterio }\end{array}$ \\
\hline Criterio 1 & 7,9 & 8,3 & 7,8 & 7,3 & 8,3 & 9,0 & 9,0 & 8,0 & 7,8 & 8,2 & 8,2 \\
\hline Criterio 2 & 6,5 & 8,3 & 7,8 & 8,0 & 7,2 & 8,7 & 8,9 & 8,2 & 8,6 & 8,3 & 8,1 \\
\hline Criterio 3 & 8,5 & 8,4 & 6,3 & 6,4 & 8,0 & 9,4 & 9,3 & 8,6 & 9,0 & 8,3 & 8,2 \\
\hline Criterio 4 & 8,0 & 8,2 & 7,9 & 7,7 & 8,3 & 9,4 & 8,7 & 7,8 & 8,8 & 8,2 & 8,3 \\
\hline Criterio 5 & 8,9 & 8,2 & 6,6 & 7,9 & 8,0 & 9,0 & 8,7 & 8,6 & 8,8 & 8,5 & 8,1 \\
\hline Criterio 6 & 8,1 & 8,1 & 7,6 & 7,9 & 7,7 & 8,7 & 8,9 & 7,8 & 8,6 & 7,8 & 8,1 \\
\hline $\begin{array}{l}\text { Promedio } \\
\text { del residente }\end{array}$ & 7,98 & 8,3 & 7,3 & 7,5 & 7,9 & 9,0 & 8,9 & 8,2 & 8,6 & 8,2 & 8,2 \\
\hline
\end{tabular}

Tabla IV. Listado de desempeño comunicacional.

\begin{tabular}{|c|c|c|}
\hline $\begin{array}{l}\text { Posición } \\
\text { en la escala }\end{array}$ & $\begin{array}{l}\text { Promedio } \\
\text { obtenido }\end{array}$ & $\begin{array}{l}\text { Identificación del } \\
\text { médico residente }\end{array}$ \\
\hline $1 . \stackrel{0}{-}$ & 9,0 & 6 \\
\hline 2.. & 8,9 & 7 \\
\hline 3.. & 8,6 & 9 \\
\hline 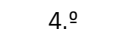 & 8,3 & 2 \\
\hline 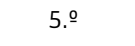 & 8,2 & 8 \\
\hline $6 . \stackrel{0}{ }$ & 8,2 & 10 \\
\hline 7.. & 7,98 & 1 \\
\hline $8 . \stackrel{\circ}{-}$ & 7,9 & 5 \\
\hline 9.9 & 7,5 & 4 \\
\hline 10. & 7,3 & 3 \\
\hline
\end{tabular}

puestos a las críticas de forma y fondo (argumentos adecuados) realizadas por los concurrentes. No existían disociaciones o disonancias cognitivas o actitudinales entre los primeros casos presentados y los últimos; muy al contrario, se optimizaba la competencia durante la exposición oral del caso clínico.

La novedad en esta investigación fue usar un método de presentación real de un caso clínico ya solucionado y no comparar con ningún otro método de evaluación de un médico residente, como las simulaciones de casos clínicos [2] .
Para establecer si el uso de esta herramienta calificadora era susceptible de usarse en otros grupos o momentos, se buscó la consistencia mediante su estimación con la prueba $\alpha$ de Cronbach, que comprobó que este método era fiable y aceptable y, por lo tanto, un instrumento preciso y válido [7] para la evaluación de una presentación oral clínica. Es un elemento calificador que proporciona apreciaciones de distintas habilidades combinadas para un objetivo y un cálculo con el menor sesgo posible, que favorece el aprendizaje de competencias y la consolidación de éstas.

Si bien es de fácil uso, su contenido debe ser supervisado por los docentes participantes antes de la presentación para estimar el proceso formador de enseñanza-aprendizaje y actuar de forma clara y precisa como un tutor que guía no solamente en técnicas quirúrgicas evaluables, sino en la manera de comunicarlas.

Los cursos basados en la práctica clínica [10] ofrecen una excelente oportunidad de aprendizaje y tienen la ventaja de ser una transición exitosa entre el papel del residente y la inminencia de una competencia comunicacional con su actitud profesional. Se evalúa de esta manera una transferencia del aprendizaje a un futuro lugar de trabajo donde el caso clínico es una herramienta fundamental en la atención sanitaria personalizada. La evaluación observacional directa permite adquirir experiencia en exponer un caso clínico, jerarquizando de forma concreta sus datos principales, y considerar el contexto específico de las posibilidades terapéuticas ya realizadas.

Usar esta forma de evaluación para la presentación de un caso clínico real facilita la evaluación do- 
cente y permite establecer las áreas donde el médico residente, como alumno, necesita más apoyo. Constituye un aprendizaje basado en tareas donde los casos en los que los residentes trabajan en un contexto real práctico se apoyan en una reflexión científico-técnica actualizada por la mejor evidencia científica. Se podrá admitir entonces las áreas a fortalecer mediante lecturas de textos, artículos o solicitando al médico docente demostraciones de otros casos clínicos. Además, se elimina la incertidumbre que posee el médico residente sobre la forma en que es evaluado. El ambiente educativo no ha de ser hostil y debe demostrarse honestidad en la presentación; si es necesario, analizar los errores para prevenir su repetición, con exposición de casos recientes que obliguen a todo el plantel del servicio de cirugía (jefes, médicos de planta, residentes, incluso alumnos de grado) a una periodicidad y obligatoriedad de concurrencia con el fin de cumplir los objetivos educativos perseguidos en el programa de residencia.

Como claras desventajas están la subjetividad si existe un único evaluador, la distorsión de los cuestionarios y los sesgos de selección o interpretación que originan errores de evaluación, que se subsanan cumpliendo los criterios preestablecidos previamente enunciados.

La limitación de esta investigación, y por ende su debilidad, es que no hay análisis semejantes con las características aquí mencionadas (presentación oral de caso clínico en reuniones asistenciales) en la bibliografía en educación médica: los resultados aquí obtenidos pueden servir exclusivamente a la cohorte observada. Asimismo, no se hizo en la misma cohorte un nuevo análisis evaluado por otros docentes debido a la falta de preparación docente de los médicos de planta asistentes a la reunión del servicio asistencial. Tampoco se evaluó en esta in- vestigación si los médicos residentes preferían este método de evaluación al no efectuarse una encuesta de satisfacción.

En conclusión, la presentación oral de un caso clínico por médicos residentes de cirugía en una reunión asistencial es un instrumento con un elevado grado de validez y fiabilidad debido a los criterios preestablecidos para una evaluación objetiva y efectiva, aunque el modelo aquí propuesto sea uno de experimentación en un mismo grupo de médicos residentes. Nuevos estudios en grupos mayores y de otras instituciones asistenciales (estatales y privadas) quizá logren ratificar lo aquí demostrado.

\section{Bibliografía}

1. Arribalzaga EB, Algieri RD, Aparicio MJ, Borracci RA, Ferrantes MS, Jacovella PF, et al. Comunicación básica en cirugía. Buenos Aires: Dunken; 2016.

2. Rodríguez-Díez MC, Beunza JJ, López-Del Burgo C, Hyder O, Civeira-Murillo MP, Díez N. Aprendizaje de la historia clínica con pacientes simulados en el grado de Medicina. Educ Med 2012; 15: 47-52.

3. Borracci RA, Arribalzaga EB. Estilos de aprendizaje de Kolb en estudiantes de grado de medicina. Medicina (B Aires) 2015; 75: 73-80.

4. Palés-Argullós JL, Gomar-Sancho C. El uso de las simulaciones en educación médica. TESI 2010; 11: 147-69.

5. Borracci RA, Arribalzaga EB. Análisis de los instrumentos de evaluación. Revista Argentina de Cirugía 1994; 66: 52-7.

6. Valenzuela $\mathrm{CH}$. Entrenamiento y evaluación del cirujano en formación. 84. ${ }^{\circ}$ Congreso Argentino de Cirugía. Revista Argentina de Cirugía 2013; 104: 1-34.

7. Hernández-Sampieri R, Fernández-Collado C, Baptista-Lucio P. Metodología de la investigación. México DF: McGraw-Hill Interamericana; 1998.

8. Hales B, Terblanche M, Fowler R, Sibbald W. Development of medical checklist for improved quality of patient care. Int J Qual Health Care 2008; 20: 22-30.

9. Hepp KJ, Csendes A, Ibáñez F, Llanos O, San Martín S. Programa de la especialidad Cirugía General. Definiciones y propuestas de la Sociedad de Cirujanos de Chile. Revista Chilena de Cirugía 2008; 60: 79-85.

10. Sinha SN, Page W. Interns' day in surgery: improving intern performance simulation-based course for final year medical students. ANZ J Surg 2015; 85: 27-32. 